\title{
Implementation and Evaluation of a Traffic Light Assistance System Based on V2I Communication in a Simulation Framework
}

\author{
Cristina Olaverri-Monreal (iD, ${ }^{1}$ Javier Errea-Moreno $\left(D,{ }^{2}\right.$ and Alberto Díaz-Álvarez ${ }^{3}$ \\ ${ }^{1}$ Department of Information Engineering and Security, University of Applied Sciences Technikum Wien, Vienna, Austria \\ ${ }^{2}$ Department of Electrical and Electronic Engineering, Universidad Pública de Navarra, Pamplona, Navarra, Spain \\ ${ }^{3}$ Universidad Politécnica de Madrid, Instituto Universitario de Investigación del Automóvil (INSIA), Madrid, Spain
}

Correspondence should be addressed to Cristina Olaverri-Monreal; olaverri@technikum-wien.at

Received 30 October 2017; Accepted 8 February 2018; Published 18 March 2018

Academic Editor: Yuchuan Du

Copyright (C) 2018 Cristina Olaverri-Monreal et al. This is an open access article distributed under the Creative Commons Attribution License, which permits unrestricted use, distribution, and reproduction in any medium, provided the original work is properly cited.

\begin{abstract}
Cooperative Intelligent Transportation Systems (C-ITS) make the exchange of information possible through cooperative systems that broadcast traffic data to enhance road safety. Traffic light assistance (TLA) systems in particular utilize real-time traffic light timing data by accessing the information directly from the traffic management center. To test the reliability of a TLA system based on networked intervehicular interaction with infrastructure, we present in this paper an approach to perform theoretical studies in a lab-controlled scenario. The proposed system retrieves the traffic light timing program within a range in order to calculate the optimal speed while approaching an intersection and shows a recommended velocity based on the vehicle's current acceleration and speed, phase state of the traffic light, and remaining phase duration. Results show an increase in driving efficiency in the form of improvement of traffic flow, reduced gas emissions, and waiting time at traffic lights after the drivers adjusted their velocity to the speed calculated by the system.
\end{abstract}

\section{Introduction}

Cooperative Intelligent Transportation Systems (C-ITS) make the exchange of information possible through cooperative systems that broadcast traffic data to enhance road safety. Lab-controlled platforms provide the test bed conditions required to perform realistic experiments with massive amounts of valuable data. This allows for the evaluation of a variety of protocols, as well as interaction with in-vehicle systems and services [1].

According to research developed in the United States by the National Highway Traffic System Administration (NHTSA), drivers who did not respect red lights in an intersection controlled by a traffic light caused from 1997 until 2004 an average of $51 \%$ of the fatal traffic crashes [2].

The technology behind traffic light timing programs is rapidly evolving. The aim is to further develop adaptive signal control technology (ASCT) in an effort to adapt traffic light timing programs to the demands of real-time traffic and thus reduce traffic congestion in urban areas. However, these technologies have only been implemented in a small number of road networks, and the majority of urban areas still have pretimed control systems installed in their traffic systems. Their preset time intervals are the same every time the signal cycles, regardless of changes in traffic volume [3]. This pretimed mode is ideally suited to closely spaced intersections where traffic volumes and patterns are consistent. It can be used to provide efficient coordination with other traffic signals, since both the start and end of green light phases are predefined. On the other hand, pretimed control tends to be inefficient at intersections where traffic arrivals are random. This causes redundant and unnecessary waiting times, significant traffic jams, and excessive delays [4].

Some systems use different preset time intervals for morning rush hour, evening rush hour, and other busy times based on historical data. Even if the information of the traffic lights is stored at each traffic management center, it is not yet 
used to help drivers to improve their driving behavior while approaching an intersection controlled by a traffic light [6] Some works have demonstrated that visual warning signals can improve driving behavior [7], and due to the complex traffic situations at urban intersections there is a need for more intelligent control solutions.

Along this line of intelligent solutions, vehicle-toeverything communication (V2X) is applied to Intelligent Transportation Systems (ITS) to establish not only vehicleto-vehicle (V2V) connection, but also to the surrounding infrastructure. This connectivity enhances traffic situational awareness across the entire road network and aims at optimizing traffic flow, reducing the number of road accidents, lowering traffic congestion, and minimizing carbon emissions. V2X technologies require in-vehicle technology (i.e., on-board units) and roadside units (RSU) attached to the infrastructures of the road. The world's first digital highway was established in 2015 on the A9 highway in Bavaria, Germany [8]. Digital highways are stretches of road for introducing and testing applications based on V2X. More than 50 car companies, such as Audi, Mercedes, and BMW, have expressed their interest in testing new technologies on this digital highway.

ITS provide real-time information for decision-making algorithms that can be then used by travelers and traffic management systems. Different advanced driver assistance systems (ADAS) have been developed to make drivers aware of their surroundings while driving. Some of the most common include forward collision warning (FCW), which detects a leading vehicle and calculates relative speed and distance in order to generate warning signals based on time to collision (TTC); traffic sign recognition (TSR) or systems that recognize different traffic signs to augment the field of view of the driver; traffic light recognition (TLR), to detect and recognize traffic lights; and traffic light assistance (TLA) systems that acquire data related to the signal timing, tailback, and geometry of intersections and combine it with in-vehicle data [9]. For each of them, collection of different data is required, such as speed or distance to the following car or next traffic light.

As a natural advance in the research in cooperative systems and automated vehicle development, autonomous driving technology includes V2X capabilities. We implemented a simulator platform to test those ADAS that rely on vehicle-to-infrastructure- (V2I-) based communication and present in this paper a TLA system in a simulated context. The proposed system retrieves the traffic light timing program within a range, in order to calculate the optimal speed while approaching an intersection. The system warns the driver by displaying a message in the dashboard, showing a recommended velocity based on the vehicle's current acceleration and speed, phase state of the traffic light, and remaining phase duration. Driving efficiency is increased once the driver adjusts their velocity to the speed calculated by the system. By retrieving the information from the traffic light predefined program, it is possible to improve traffic flow and reduce traffic delays.

In order to develop the approach, Simulation of Urban Mobility (SUMO) [10], Unity 3D [11], and City Engine [12] were used for the development of the two different scenarios for the experiments. Routing directions were displayed in the user-controlled vehicle (UCV) in both scenarios.

Scenario 1. UCV had the TLA system activated.

Scenario 2. UCV did not have the TLA system activated.

We defined the following research questions that were tested within the described framework.

(1) Is there a significant amount of time saved at the light, waiting, and as a consequence travel time from start to destination, by implementing and using TLA technology?

(2) Do TLA systems improve the efficiency of traffic flow?

(3) Do TLA systems reduce the amount of $\mathrm{CO}_{2}$ emissions?

We additionally define the following null hypothesis:

H0: the TLA system that conveys the optimal velocity for approaching an intersection does not reduce the time waiting at a traffic light or the total time spent to reach the destination, nor does it result in an improvement of traffic flow.

The next section considers related work in simulation approaches. Section 3 describes the development of the simulation platform. Section 4 presents the implementation of the TLA system. Section 5 describes the experimental procedure to test the TLA. Section 6 reports on the performance and validation results of the developed system. Finally, Sections 7 and 8 discuss the results and conclude the paper.

\section{Related Work}

Several works have reported on the application of TLA services based on C-ITS standards. For example, the authors in [14] describe some significant steps for cooperative signaling that involve connecting to the city infrastructure to generate the service data. They argue that, in most European cities, traffic light controllers are equipped with inadequate hardware and do not allow proper communication with the traffic management center.

The authors in a further work [9] present and test a traffic light assistant prototype that combines V2X and mobile communication in real traffic. This TLA prototype extracts signal timing, tailback, geometry, and hazard information of intersections and combines it with internal vehicle data. Concepts for processing, management, and visualization of V2X and Traffic Signal Information (TSI) messages are also described.

In order to test $\mathrm{V} 2 \mathrm{~V}$ and $\mathrm{V} 2 \mathrm{I}$ application developments in a controlled system, [15] combines a traffic and communication simulator with a multiuser driving simulator for a low-cost testing environment. In line with this research, we present in this paper an approach that simulates a visual TLA system in Unity 3D, using SUMO as the traffic simulator in order to test the research questions and hypotheses defined in the previous section. 
Car manufacturers have already announced the first traffic light information systems based on V2I communication through 4G LTE connection [16]. The first real life experiments with V2I services were carried out at the end of 2017.

Regarding the effectiveness of the output modality to convey information related to traffic lights, both visual and auditory warnings have been studied in simulation frameworks. Most of these studies focused on visual warnings for displaying the messages created by the system. In [7], five different human machine interfaces (HMI) were evaluated. Each of the HMI provided the same information that was displayed in various manners. The study concluded that all the HMI contributed to augmenting situational awareness regarding the phase of the traffic light.

A further study that focused on conveying information based on TTC regarding a vehicle running an upcoming red light stated that visual warnings distracted drivers [17]. However, the relationship between auditory warning systems and driving behavior is still ambiguous and requires further investigation, although many visual ADAS systems are already integrated into vehicles and fully accepted by drivers.

TLA systems utilize real-time traffic light timing data by accessing the information directly from the traffic management center and combining it with GPS data. This makes it possible for vehicles to calculate exactly which traffic light they are approaching, whether that light is red or green, and how long it will be until it changes. The vehicle also calculates if it will be able to drive through an intersection with a green traffic light based on how fast it is moving. All of these data alert the drivers of any upcoming situations posed by the next intersection and help them to adjust their speed in order to avoid waiting at red lights.

Evaluation of new in-vehicle technology using simulators has been performed in different works. For example, in [18] a user interface for a novel traffic regulation system was assessed. The interface conveyed information to the driver which was based on the ubiquitous optimized management of individual intersections where physical devices were replaced by virtual traffic lights (VTL). However, only a few works address in-vehicle technology based on simulated environments with V2I capabilities, and as far as it is known to the authors, no approach of a visual TLA system has been implemented in Unity 3D using SUMO as the traffic simulator.

\section{Simulation Framework Implementation}

3.1. Generation of the Scenario Network. Using the traffic network defined in the XML SUMO network file, a complex network was created to be used as the scenario of the simulation. SUMO uses an $X-Y$ coordinate system to locate all the elements of the network. The selected area was the Neubaugurtel Strasse in Vienna, as the amount of traffic lights on this road is significant. The source used for extracting data was Open Street Map (OSM) [19] since it allows the selection of areas of interest and automatically generates an .osm file. The desired area was defined manually as shown in Figure 1. In order to create the corresponding .net.xml file,

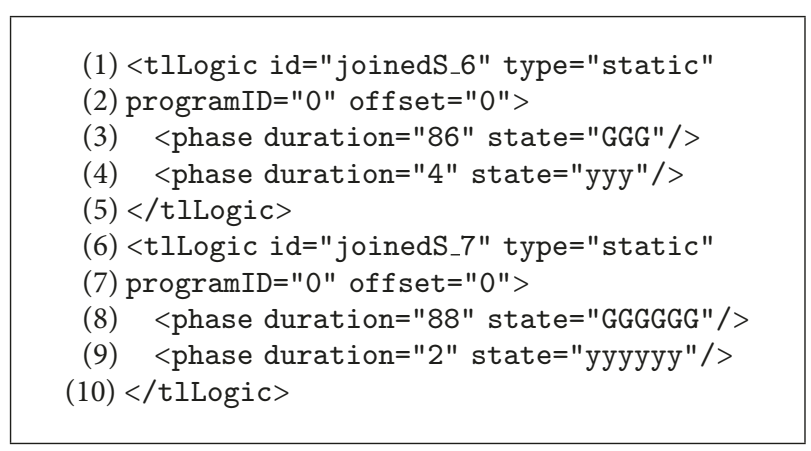

Algorithm 1: Errors in the TLS program.

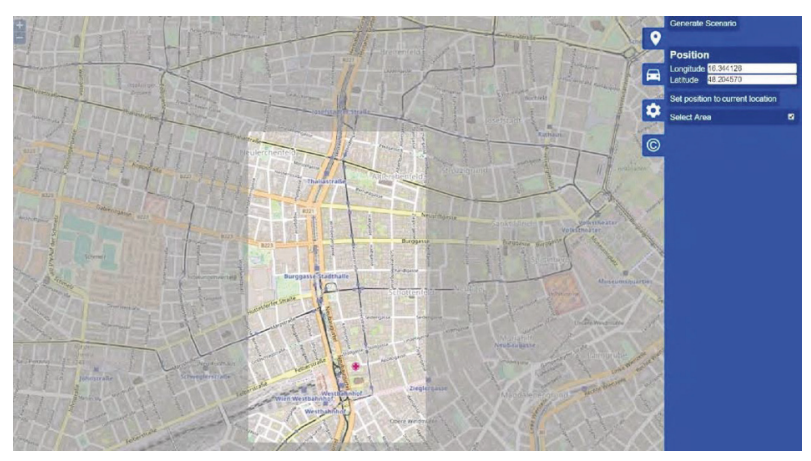

FIGURE 1: Definition of the area from Open Street Map used in the experimental scenario.

the application NETCONVERT was used. Additional data included in OSM regarding buildings, parks, rivers, and so forth was visualized by using the POLYCONVERT tool.

To edit the network the tool NETEDIT was used, and the junctions in which there were a significant number of intersections were merged. Connections between roads were also edited.

3.2. Modification of TLS Programs for the Created Network. The .net.xml file generated from the NETCONVERT tool includes information about the traffic light systems (TLS). After verifying the .net.xml file, it was found that not all of the TLS programs with only one intersection had a red phase. An example of these errors can be found in the code in Algorithm 1.

In addition, some phases were longer than in reality. Therefore, this behavior was adapted for real conditions by shortening the length of the phases and/or adding missing red light phases. Once these two modifications were done, the edition of the network was completed.

3.3. Connection of SUMO with Unity $3 D$ Using the TraCI Protocol. In order to create a connection between the SUMO and Unity 3D simulators, an interface was required to implement the communication. The communication protocol Traffic Control Interface (TraCI) was used to this end. TraCl's architecture is Client-to-Server, so that information that is required dynamically can be accessible to both SUMO and Unity 3D. SUMO acts as a TraCI server and it will listen 
for incoming connections from external applications. Once SUMO has accepted the connection request, the TraCI client takes control of the simulation, which enables the client to affect the behavior of the simulation by sending commands to SUMO. As described in [20], in order to implement the $\mathrm{TraCI}$ protocol the existing library $\mathrm{TraCI}$ as a Service (TraaS) [21] was selected, as it allows remote control of SUMO from any programming language.

3.4. Scenario Set-Up. The Unity project created to evaluate the implemented TLA consisted of a set of folders, such as prefabs (vehicles and roads models), scripts, materials (characterization of models), Plug Ins (data base connection), and $\mathrm{TraCI}$ library (implementation of TraCI protocol). These folders were located in the Assets folder to be read.

As previously mentioned, we created an urban scenario to test the use cases with and without the TLA system implemented. Once the scenario was initialized, information about the simulated objects of interest such as buildings and their shape was retrieved. Additionally, real tree data were downloaded from the Vienna Municipal Departments 41 [22] and 42 and then imported into CityEngine as an object file, which was used to create the experiment's urban environment. An additional script was implemented to apply textures to the objects imported to CityEngine. Key buildings were thus recognized by the participants.

All the described parts constituted the final scenario that was imported as an .fbx file into Unity 3D as a GameObject. Once the urban environment was completed, a Mini Cooper vehicle was imported into the Unity 3D game engine and defined through scripts that also described the steering wheel and wheel movement.

\section{Traffic Light Assistance Implementation}

Vehicles in SUMO automatically follow a defined TLS schedule. In order to represent the traffic lights in Unity 3D, we used models of traffic lights and created a script to manipulate their behavior. Traffic lights are not defined as such in SUMO. Instead, SUMO uses TLS to control specific intersections. Consequently, one intersection might have more than one traffic light within a single TLS. Therefore, traffic lights were placed manually in their corresponding positions, and timing was adjusted in accordance with the TLS's schedule in SUMO.

We implemented a script that contained a matrix of Boolean variables describing the different modes of the traffic light. Each mode was defined by three variables, one per state, that were ordered as follows: red-yellow-green. Depending on the actual phase of the traffic light, the variable duration was modified according to the phase duration in SUMO. For changing the color of the traffic light, the three variables of the actual mode were verified. The color was set depending on which position of the matrix was true. The code in Algorithm 2 shows an example in which the traffic light state is green.

A TLA ADAS system was created and integrated into a UCV. This system computes the Green Light Optimal Speed Advisory (GLOSA) algorithm [5] shown in Algorithm 3 in order to calculate the optimal speed for approaching the next traffic light.

In order to compute this algorithm, several integral components of information about the TLS and the UCV were required. The information collected for each TLS was as follows:

(i) Time until next green phase.

(ii) Current state of the TLS.

(iii) Complete GreenYellowRed program of the TLS.

(iv) Links controlled by the TLS.

SUMO defines a fixed-time program for each TLS, and it can be either static or actuated. TLS programs describe the behavior of all the traffic lights placed at an intersection. For example, in the following state definition of a TLS phase, $<$ phaseduration $=25$ state $=R R g g g g R r />$ each character in the string within a phase's state describes the state of one link of the TLS ( $\mathrm{g}$ for green, $\mathrm{y}$ for yellow, and $\mathrm{r}$ for red). One lane may contain more than one link, for example, one for vehicles turning right and one for vehicles continuing straight.

The state of a TLS is defined as a string of characters. Each character describes one link controlled by the TLS. When the position of the character and the lane at which the vehicle is located match, the state is verified. Then, two variables called $t$ min and $t$ max are defined. Tmin contains the minimum time until the traffic light is green, defining the exact moment at which it would change from red to green. $t$ max contains the time at which the next green phase will end.

Based on the performed calculations several messages regarding a recommended velocity for the UCV were displayed in the dash board screen. The messages displayed depended on the value returned by the TLA algorithm and the corresponding calculated velocity based on the distance of the UCV to the traffic light. If this distance was smaller than 80 meters, the GLOSA algorithm was computed and the correspondent message to reach the traffic light in the green phase from the list below was conveyed to the driver.

(i) Actual speed is optimal.

(ii) Speed needs to be decreased.

(iii) Speed needs to be increased according to the recommended range.

(iv) The system informs of a necessary stop at traffic light due to the long duration of the red phase in combination with the speed calculated by the algorithm.

Figure 2 shows one example for a message that recommends reducing velocity in order to reach the traffic light in the green phase.

\section{Experimental Procedure}

In order to test the performance of the TLA system, experiments were performed on drivers under lab-controlled conditions. After being welcomed, each participant was given a brief explanation of the tasks to perform in the simulator. 


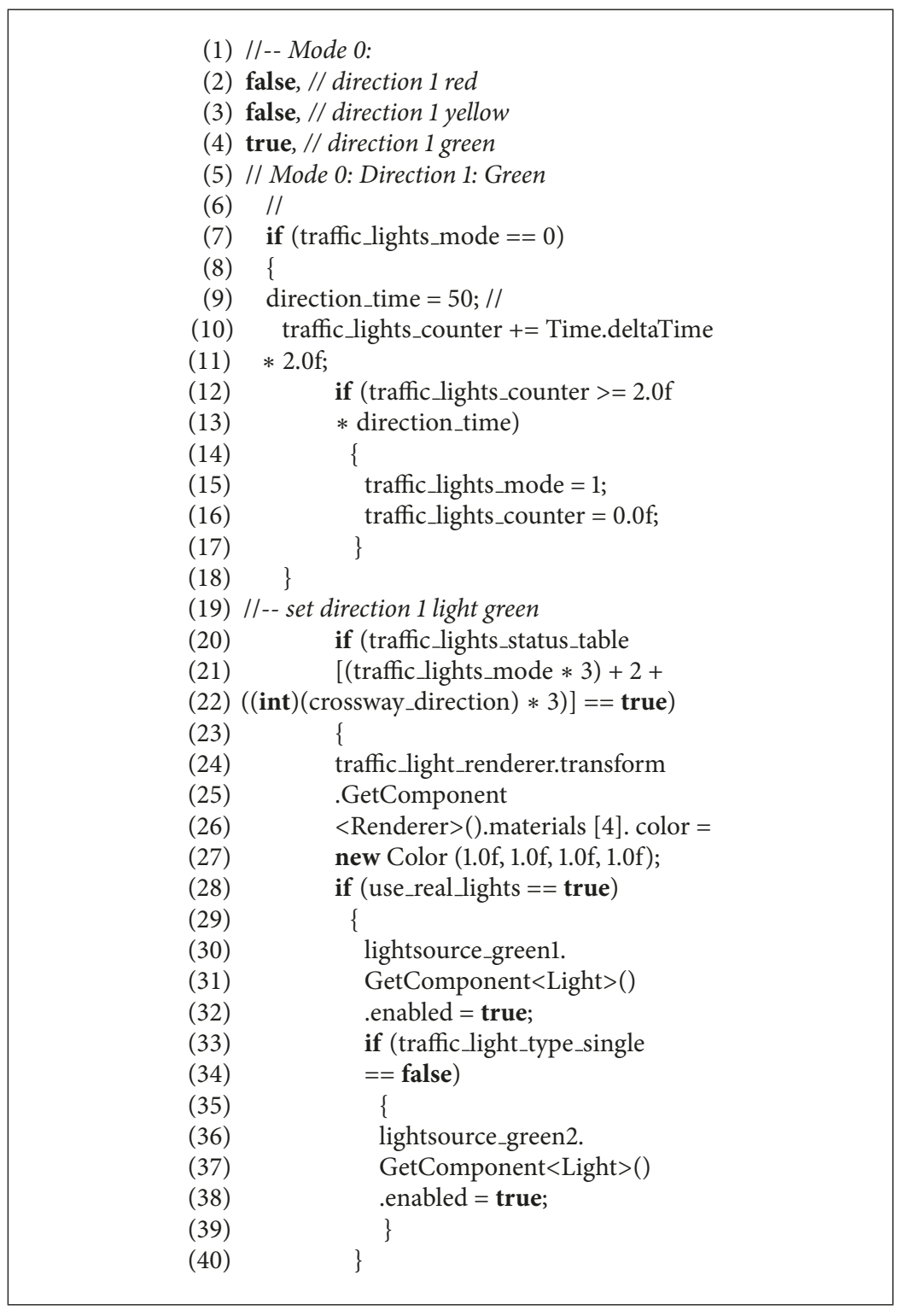

Algorithm 2: Green light algorithm.

They then drove for five minutes in order to get familiarized with the simulation platform. The flow chart of Figure 3 depicts the experimental procedure. A total of 25 participants aged between 21 and 55 completed the experiment (mean age $=27.5, \mathrm{SD}=9.2,64 \%$ male, $36 \%$ female). They were asked to follow the traffic rules and to not exceed the speed limit for an urban area of $40 \mathrm{~km} / \mathrm{h}$. All participants followed the route directions displayed on the dash board as they would with a navigation system.

In Scenario 1, the TLA ADAS system was not activated; therefore the data were logged for a representation of the baseline condition. Once the participants reached the end of the route, Scenario 2 with the activated TLA system was loaded and the corresponding data logged (see example in Figure 2). The duration of each phase was 15 minutes. When the experiment was completed, each participant was asked to fill out a questionnaire to rate their experience with the simulator and the efficiency of the system implemented. Figure 4 shows a participant during the experiment with the TLA system activated.

5.1. Data Acquisition and Processing. The pertinent data for testing the hypotheses were logged. First, the state and remaining phase time of the link at which the UCV was placed was retrieved and stored. Additional data related to the UCV regarding speed, distance, and time were logged and stored in a SQLite database. The data were then processed to calculate the main metrics from the simulation experiments: driver's average speed, total traveled time, delay or waiting time at traffic lights, $\mathrm{CO}_{2}$ emissions, and number of stops.

In order to calculate the $\mathrm{CO}_{2}$ emissions, a method was used relying on the speed-based model proposed by the National Institute for Land, Infrastructure and Transport (NILIM) [23]. This model is based on the size and weight 


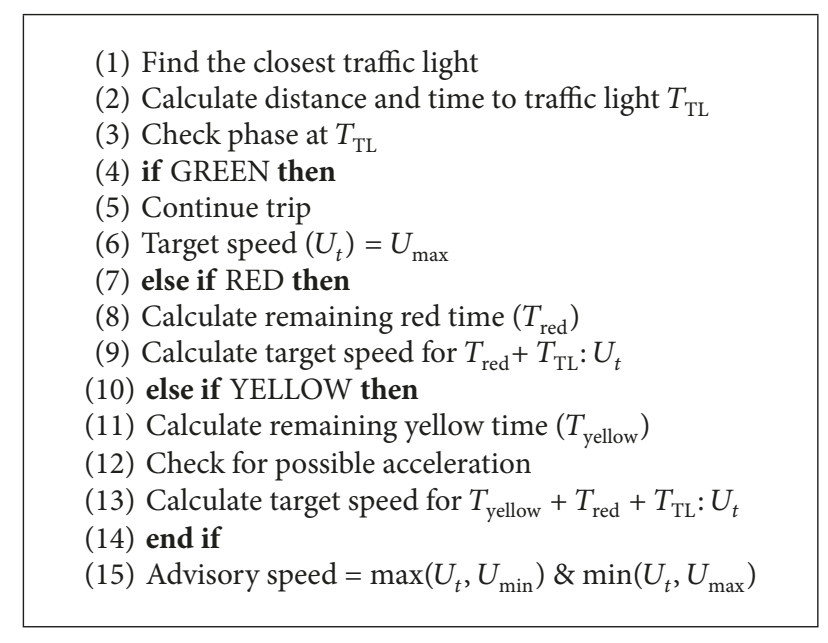

Algorithm 3: GLOSA algorithm [5].

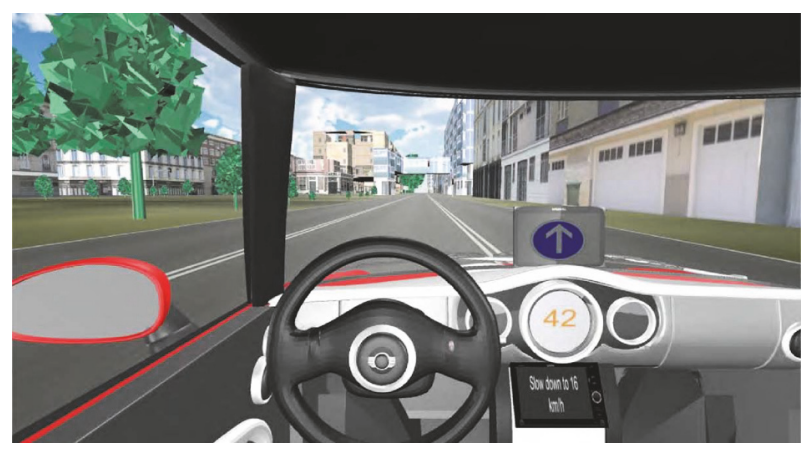

FIGURE 2: TLA system conveying information regarding a decrease of the current UCV velocity.

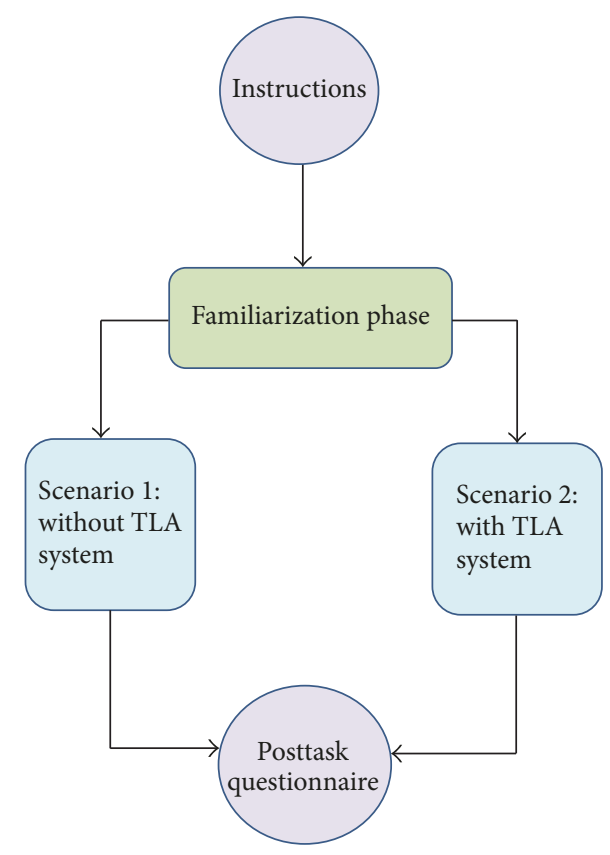

FigURE 3: Flow chart of the experimental procedure.

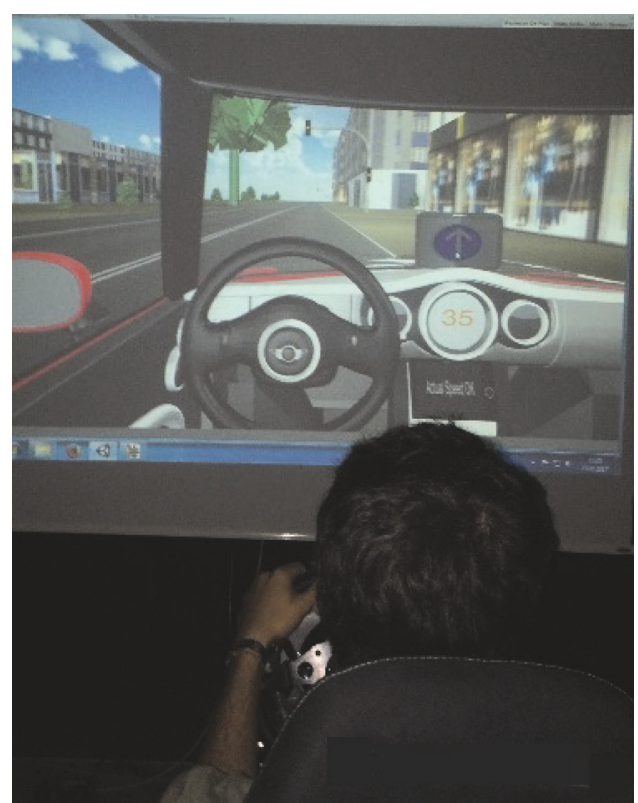

FIGURE 4: Participant performing experiment with TLA system activated.

of the vehicle in question (small and large), the average speed of the vehicle, and the distance traveled. A Mini Cooper was used as the UCV in the simulation; therefore the approximation for a small vehicle was used to determine the $\mathrm{CO}_{2}$ emissions as described in (1) [13].

(i) $\mathrm{EF}_{\mathrm{CO} 2}\left(V_{i}\right)=$ emission factor of $\mathrm{CO}_{2}$ per unit distance.

(ii) $V_{i}=$ average travel speed of vehicle $i$.

(iii) $a_{1}, a_{2}, a_{3}$, and $a_{4}=$ parameter:

$$
\mathrm{EF}_{\mathrm{CO} 2}\left(V_{i}\right)=\frac{a_{1}}{v_{i}}+a_{2} v_{i}+a_{3} v_{i}^{2}+a_{4} .
$$

The resulting equation for the total amount of $\mathrm{CO}_{2}$ emissions of a vehicle is as follows: $E \mathrm{CO} 2_{i}=\mathrm{EF}_{\mathrm{CO} 2}\left(v_{i}\right) x_{i}$, where $x$ is the total distance traveled. Figure 5 [13] shows the relationship between average speed and $\mathrm{CO}_{2}$ emissions.

An approximation of the curve for the small vehicles was written into the $\mathrm{C} \#$ program language in order to calculate the $\mathrm{CO}_{2}$ throughout the evaluation of the simulator. The variables were logged into the database.

Regarding the collection of data related to the qualitative evaluation of the system, a Likert scale was used with values that ranged as follows: $1=$ strongly disagree to $5=$ strongly agree. The questionnaire asked the participants to rate the following four elements:

(1) Effect of the TLA on the driver's behavior

(2) Satisfaction with the messages displayed by the TLA system

(3) Performance of the TLA system

(4) Distraction level of TLA 
TABLE 1: Driving performance depending on the activation of the TLA.

\begin{tabular}{|c|c|c|c|c|c|c|}
\hline & \multicolumn{2}{|c|}{ W/o TLA } & \multicolumn{2}{|c|}{ W/TLA } & \multicolumn{2}{|c|}{$T$-test $(\alpha=0.05)$} \\
\hline & Mean & $S D$ & Mean & $S D$ & $p$ & $t(24)$ \\
\hline Travel time (s) & 398 & 52.57 & 370.2 & 43.8 & 3.19 & $0.0038^{* *}$ \\
\hline Delay $(s)$ & 108.59 & 35.82 & 71.38 & 26.64 & 6.82 & $4.69 e-7^{* * *}$ \\
\hline Speed $\left(\mathrm{kmh}^{-1}\right)$ & 19.16 & 2.57 & 20.4 & 2.2 & -2.88 & $0.008^{* *}$ \\
\hline $\mathrm{CO}_{2}(\mathrm{mg})$ & 411.91 & 28.12 & 406.33 & 22.11 & 1.19 & 0.24 \\
\hline Stops no. & 13.6 & 3.88 & 8.88 & 4.4 & 5.66 & $7.69 e-6^{* * *}$ \\
\hline
\end{tabular}

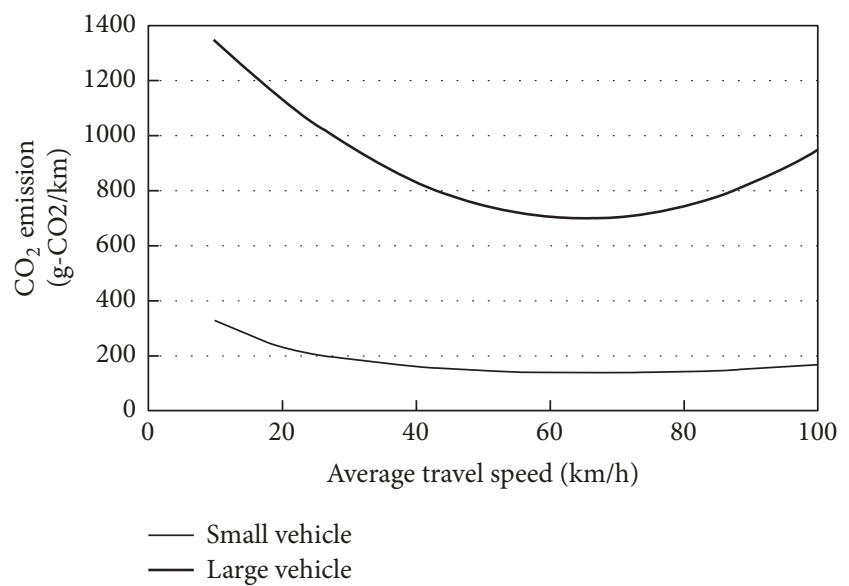

FIGURE 5: Relationship between average speed and $\mathrm{CO}_{2}$ emissions according to [13].

5.2. Data Analysis. Metrics related to driving performance were compared using the paired $t$-test to determine whether the TLA system improved driver performance and to draw conclusions about the impact of the proposed ADAS system. The test calculates the difference within each before-andafter pair of measurements, determines the means of these changes, and reports whether this mean of difference is statistically significant.

The $\alpha$ level of significance used for the performance of the $t$-test was 0.05 . The following steps were followed in order to test the validity of the null hypothesis [24]:

(i) Calculation of the sample difference mean $(\widetilde{d})$ is as follows. In order to get this value, the difference between each value of the observed paired driving metrics from the different scenarios $(X$ and $Y$ ) was calculated (see equation (2) [24]).

$$
\begin{aligned}
D_{i} & =Y_{i}-X_{i} . \\
\widetilde{d} & =\frac{1}{N} * \sum_{i=0}^{n} D_{i}
\end{aligned}
$$

(ii) Calculation of the standard deviation of the difference $\left(S_{d}\right)$ in order to calculate the standard error of the mean difference $(\mathrm{SE}(d))$ is as follows. $\mathrm{SE}(d)=S_{d} / N$, where $N$ is the strength of the sample. (iii) Calculation of the $t$-statistics $(T)$ is as follows. This calculation follows a $t$-distribution with $(N-1)$ degrees of freedom under the null hypothesis.

$$
T=\frac{d}{\operatorname{SE}(d)} .
$$

The $T$ value is compared with the $T(N-1)$ distribution using the table of the $t$-distribution, which gives the $p$ value for the paired $t$-test.

The $p$ value determines whether the results are statistically significant or not. The null hypothesis can only be rejected when the $p$ value obtained is smaller than the significance value $(0.05)$.

\section{Results}

6.1. Quantitative Evaluation. The total time traveled, average speed, total time waiting at red lights, number of stops at traffic lights, and $\mathrm{CO}_{2}$ emissions were the parameters measured during the experiments. Table 1 shows the comparison of the mean values and the standard deviation between the scenario without the TLA and the scenario with the TLA system. The average values of $\mathrm{CO}_{2}$ emissions were very similar. However, there was a slight reduction of $3 \%$ with the TLA system activated. By implementing the TLA system the total time spent completing the route was remarkably reduced. This difference in time was statistically significant with respect to the one obtained without the TLA system. Regarding the number of stops at traffic lights, 20 traffic lights were placed throughout the scenario. Without the implementation of the TLA system, the average number of stops per participant was 13.6 ( $68 \%$ of the traffic lights), whereas with the help of the TLA system, the average number of stops was reduced to $8.88(44.4 \%)$. Due to this fact, the total time spent waiting for the traffic light to change to its green phase was reduced in $34.22 \%$.

Figure 6 shows how the activation of the TLA produces an increase in the average speed and a decrease in the rest of the indicators. Figure 7 shows the efficiency improvement by comparing the variables in both scenarios.

6.2. Correlations. Correlation matrices between the monitored indicators in Scenarios 1 and 2 are shown in Tables 2 and 3. The values correspond to the Spearman correlation coefficient between each pair of variables. 


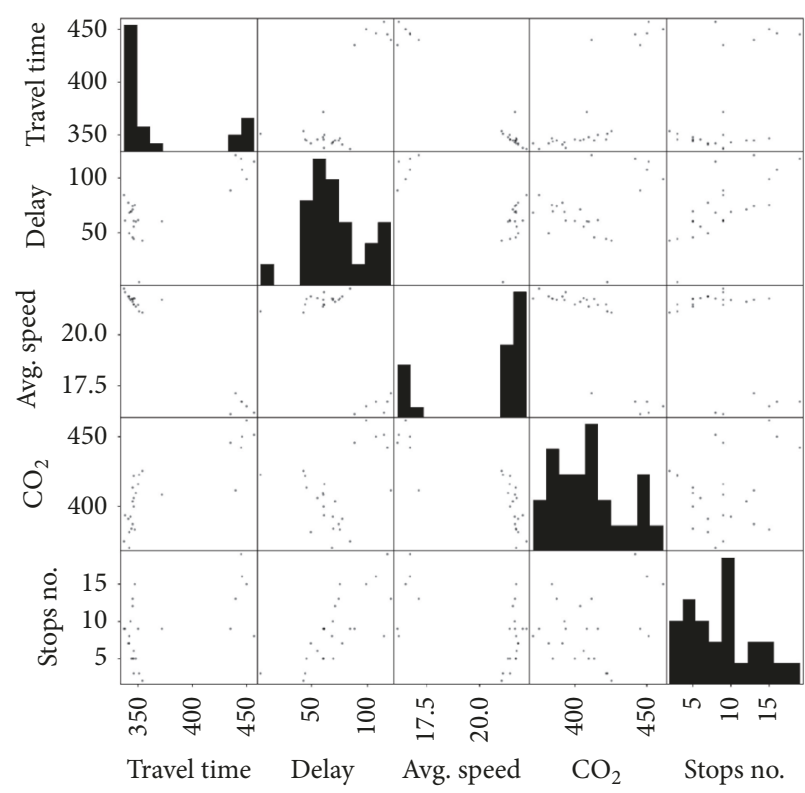

(a) Scenario 1 (with Traffic Light Assistance (TLA))

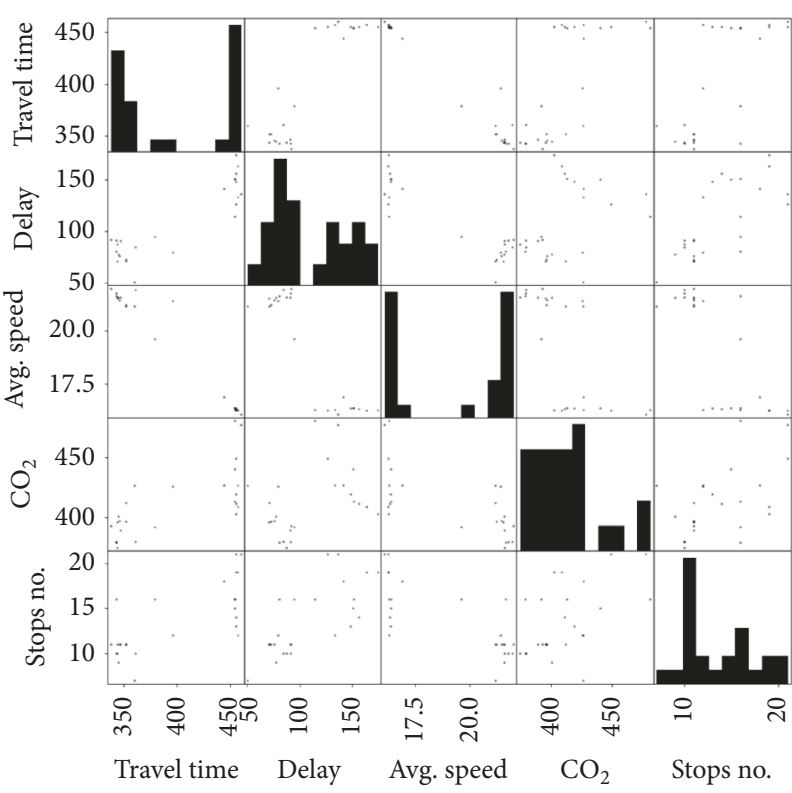

(b) Scenario 2 (without TLA)

FIGURE 6: Scatter plots for each pair of the results for both scenarios, showing the relationships between each pair of variables. The diagonal (the relationship between a variable and itself) represents the histogram of the variable on its own domain.

TABLE 2: Correlation matrix between the monitored indicators in Scenario 1.

\begin{tabular}{lccccc}
\hline & Total time & Delay & Avg. speed & CO $_{2}$ & Stops no. \\
\hline TotalTime & 1.000000 & 0.757185 & -0.986162 & 0.809822 & 0.558153 \\
Delay & 0.757185 & 1.000000 & -0.730260 & 0.341748 & -0.748499 \\
AveSpeed & -0.986162 & -0.730260 & 1.000000 & -0.820531 & 0.516156 \\
$\mathrm{CO}_{2}$ & 0.809822 & 0.341748 & -0.820531 & 1.000000 & 0.228826 \\
Stops no. & 0.558153 & 0.748499 & -0.516156 & 0.228826 & 1.000000 \\
\hline
\end{tabular}

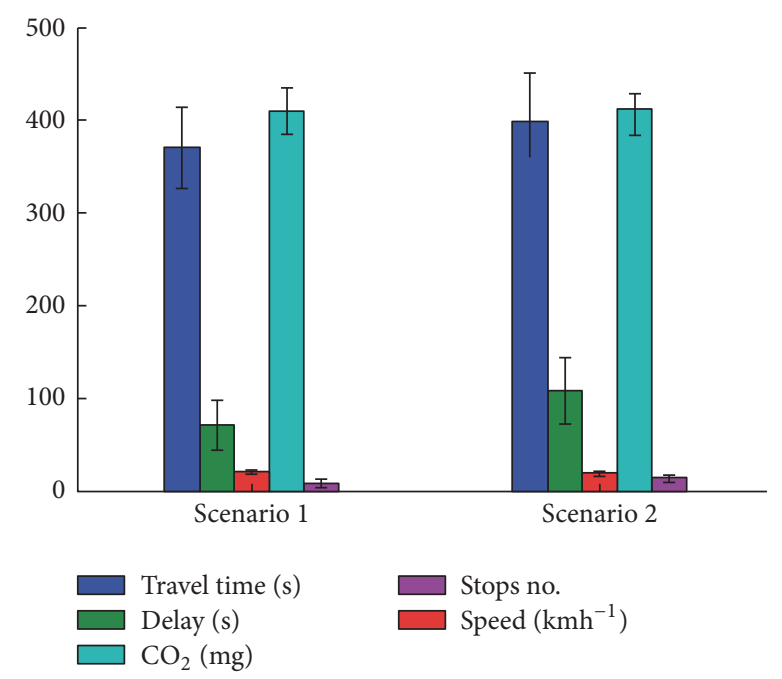

FIgURE 7: Comparison of the average variable values in Scenario 1 (with TLA) and Scenario 2 (without TLA).

Figure 8 depicts the correlations between the variables in Tables 2 (Scenario 1) and 3 (Scenario 2). Although both graphs seem to maintain similar correlations, Figure 8(a) shows a decrease in the impact of the average velocity on $\mathrm{CO}_{2}$ emissions in Scenario 1. This behavior is a consequence of the TLA activation, and it has an effect on the decrease of delays, producing on the one hand an increase in average speed and, on the other hand, more constant regimes that reduce acceleration and jerks, therefore lower emissions and fuel consumption.

6.3. Qualitative Evaluation. Results regarding the qualitative evaluation showed that almost all of the participants were able to understand the messages displayed by the system. 3 participants $(12 \%)$ did not follow the recommendations of the system because they were engaged in observing the surrounding scenario. Seven participants (28\%) felt distracted by the TLA system, the mean value obtained for the subjective distraction level being 3.08 (Likert scale range $1=$ strongly disagree to 5 = strongly agree).

The participants rated the system as helpful and useful, the mean value obtained being 4.12. A graphical representation of the ratings obtained from the postquestionnaire is shown in Figure 9. Some extra comments and possible improvements to the TLA system were recorded. For example, one participant mentioned that speed advice messages could also be triggered as audio messages instead of visual 


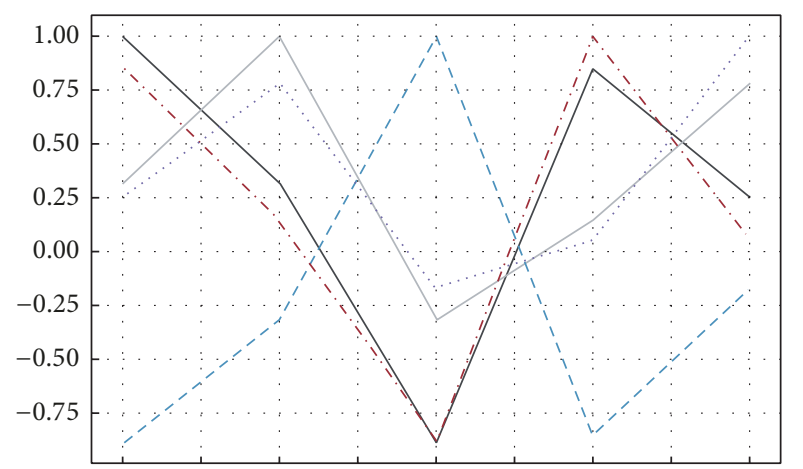

$\begin{array}{lll}\text { Travel time } & \cdots & \mathrm{CO}_{2} \\ - \text { Delay } & \ldots . & \text { Stops no. } \\ --- \text { Avg. speed } & & \end{array}$

(a) Scenario 1 (with TLA)

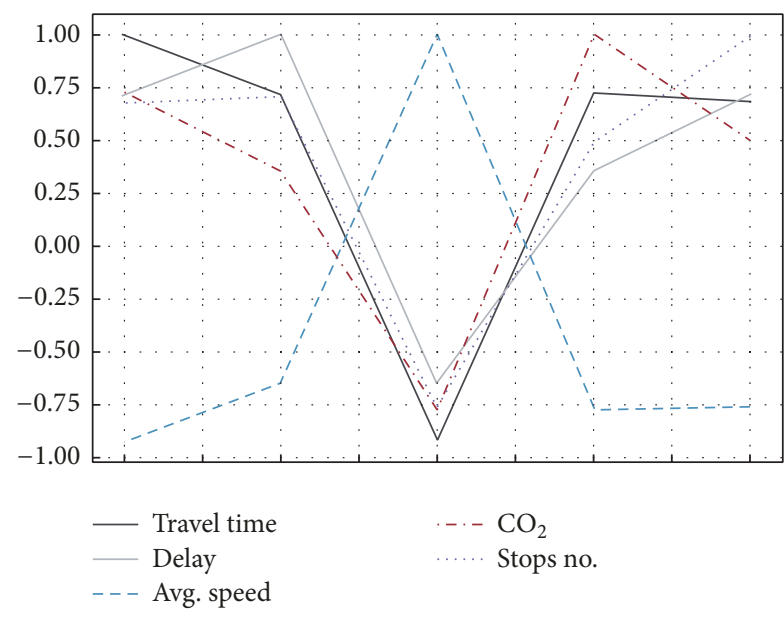

(b) Scenario 2 (without TLA)

FIGURE 8: Graphical representation of the correlations depicted in Tables 2 (Scenario 1) and 3 (Scenario 2).

TABLE 3: Correlation matrix between the monitored indicators in Scenario 2.

\begin{tabular}{lccccc}
\hline & Total time & Delay & Avg. speed & $\mathrm{CO}_{2}$ & Stops no. \\
\hline TotalTime & 1.000000 & 0.894721 & -0.980759 & 0.699022 & 0.734660 \\
Delay & 0.894721 & 1.000000 & -0.907758 & 0.383627 & 0.725905 \\
AveSpeed & -0.980759 & -0.907758 & 1.000000 & -0.675797 & -0.755984 \\
$\mathrm{CO}_{2}$ & 0.699022 & 0.383627 & -0.675797 & 1.000000 & 0.513207 \\
Stops no. & 0.734660 & 0.725905 & -0.755984 & 0.513207 & 1.000000 \\
\hline
\end{tabular}

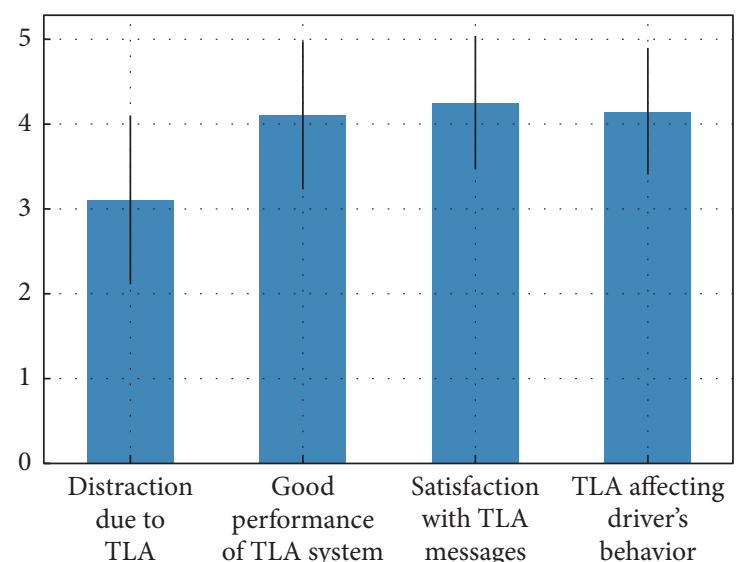

FIGURE 9: Ratings obtained from the qualitative postquestionnaire.

messages, even if the information conveyed would have to be expressed in a more complex manner. Additionally, $20 \%$ of the participants made the suggestion of placing the TLA system in a higher position, to have the messages at a more optimal point within the driver's field of view.

\section{Discussion}

The driving performance metrics measured and logged throughout the simulator experiments were total traveled time, delay or waiting time at traffic lights, average speed, $\mathrm{CO}_{2}$ emissions, and number of stops.

The travel distance for each participant was around $2.1 \mathrm{~km}$ whereas, in [5], the travel distance was less than $1 \mathrm{~km}$. The maximum speed permitted for driving was $40 \mathrm{~km} / \mathrm{h}$ as the scenario was developed in an urban area. Drivers were focused on not exceeding this limit. At the beginning of the experiments, participants were familiarized with the pedals and steering wheel and became accustomed to the manner in which the messages that signaled the route were presented.

The mean number of stops without the TLA activated was significantly higher than with the system activated, the TLA system thereby having a significant impact on the drivers behavior.

With the activation of the TLA system, the waiting time or delay was drastically reduced by $34 \%$. Previous literature showed a reduction of $30 \%$ [15]. Additionally, the total time of the journey was reduced because participants were able to avoid red lights. The total travel time was reduced by 9\% when using the TLA system, meaning a shorter time than in [15]. The difference in the number of traffic lights, length of the path, and maximum permissive speed in both experiments were the factors which influenced the difference in total traveled time.

There was an interrelation between the travel time and the average velocity. With the implementation of the TLA system, driving performance metrics like waiting time at traffic lights or delay and total travel time were reduced in 
comparison with the scenario without the proposed system, which resulted in an increase in the average speed.

Results regarding $\mathrm{CO}_{2}$ emissions showed that the variation was proportional to the difference between the mean speeds obtained. Similar results were obtained in [15] regarding $\mathrm{CO}_{2}$ and average speed.

The purpose of the postquestionnaire was to confirm that the proposed system was suitable for further experiments. Based on the opinion of the participants, the experience with the system was very satisfactory. The lowest value within the questionnaire was obtained from the level of distraction caused by the system. However, participants considered the messages clear and intuitive.

\section{Conclusion and Future Work}

We presented in this work the design and implementation of an in-vehicle TLA system which provides speed advice regarding optimal velocity when approaching an intersection controlled by a traffic light. In order to implement the TLA system, a driving simulator was developed. This simulation consisted of software components based on SUMO and Unity $3 \mathrm{D}$.

The defined hypothesis was tested and according to the results, the alternative hypothesis $H 1$ : the TLA system that conveys the optimal velocity for approaching an intersection reduces the time waiting at a traffic light and the total time spent to reach the destination and also improves traffic flow was accepted.

The validation of the simulator was achieved through the high participant ratings. They did not find the system irritating and they described it as useful and helpful. In conclusion, the proposed system had positive effects on the drivers' behavior and its performance was satisfactory.

The potential of TLA systems has been demonstrated in this work. However, opportunities for extending the scope of this work beyond its current state remain. The UCV retrieves the data directly from the control management center that maintains control of the operation of the traffic lights. The development of a new scenario with traffic lights and attached RSU in order to directly communicate with vehicles will follow in future work. To this end systems that enable the preparation and execution of V2X simulations (i.e., VSimRTI [25]) can be used to take advantage of existing libraries to communicate with SUMO.

\section{Conflicts of Interest}

The authors declare that they have no conflicts of interest.

\section{Acknowledgments}

This work was supported by the "KiTSmart Project City of Vienna Competence Team for Intelligent Technologies in Smart Cities," Project no. 18-07 funded by national funds through the MA 23, Urban Administration for Economy, Work and Statistics, Vienna, Austria, and the EU Erasmus + framework program.

\section{References}

[1] C. Olaverri-Monreal, "Autonomous vehicles and smart mobility related technologies," Infocommunications Journal, vol. 8, no. 2, pp. 17-24, 2016.

[2] R. Subramanian and L. Lombardo, "Analysis of fatal motor vehicle traffic crashes and fatalities at intersections, 1997 to 2004," Tech. Rep., 2007, https://crashstats.nhtsa.dot.gov/Api/Public/ ViewPublication/810682.

[3] V. Astarita, V. P. Giofrè, G. Guido, and A. Vitale, "The use of adaptive traffic signal systems based on floating car data," Wireless Communications and Mobile Computing, vol. 2017, Article ID 4617451, 13 pages, 2017.

[4] USDOT, “Traffic signal timing manual," 2017, https://ops.fhwa .dot.gov/publications/fhwahop08024/chapter5.htm.

[5] K. Katsaros, R. Kernchen, M. Dianati, and D. Rieck, "Performance study of a Green Light Optimized Speed Advisory (GLOSA) application using an integrated cooperative ITS simulation platform," in Proceedings of the 7th International Wireless Communications and Mobile Computing Conference (IWCMC '11), pp. 918-923, Istanbul, Turkey, July 2011.

[6] C. Sommer, R. German, and F. Dressler, "Bidirectionally coupled network and road traffic simulation for improved IVC analysis," IEEE Transactions on Mobile Computing, vol. 10, no. 1, pp. 3-15, 2011.

[7] M. Krause and K. Bengler, "Traffic light assistantevaluation of information presentation," Advances in Human Aspects of Road and Rail Transportation, p. 166, 2012.

[8] H. Breuer, "Urban mobility: radars key role on smart roads," 2017, https://www.siemens.com/innovation/en/home/ pictures-of-the-future/mobility-and-motors/urban-mobilityradar-technology-for-highways.html.

[9] B. Bernais, A. Lotz, and H. Pu, "Design and implementation of a traffic light assistance system based on c2x and tsi messages," in Proceedings of the AmE 2016-Automotive meets Electronics; 7th GMM-Symposium, pp. 1-6, 2016.

[10] SUMO, "DLR - Institute of Transportation Systems - SUMO Simulation of Urban MObility," 2016, http://sumo.dlr.de.

[11] Unity Technologies, "Unity - fast facts," 2016, https://unity3d .com/public-relations.

[12] Esri, "Esri cityengine - 3d modeling software for urban environments," 2017, http://www.esri.com/software/cityengine.

[13] K. Tsukui, H. Hanabusa, H. Oneyama, Y. Oshino, and M. Kuwahara, "CO2 and noise evaluation model linked with traffic simulation for a citywide area," International Journal of Intelligent Transportation Systems Research, vol. 7, no. 1, pp. 5965, 2009.

[14] M. Zweck and M. Schuch, "Traffic light assistant: Applying cooperative ITS in European cities and vehicles," in Proceedings of the 2013 2nd IEEE International Conference on Connected Vehicles and Expo, ICCVE 2013, pp. 509-513, Las Vegas, NV, USA, December 2013.

[15] K. Gajananan, S. Sontisirikit, J. Zhang et al., "A cooperative ITS study on green light optimisation using an integrated traffic, driving, and communication simulator," in Proceedings of the 36th Australasian Transport Research Forum, ATRF 2013, October 2013.

[16] A. USA, "Audi announces the first vehicle to infrastructure (v2i) service - the new traffic light information system," 2017, https://www.audiusa.com/newsroom/news/press-releases/2016/ 08/audi-announces-first-vehicle-to-infrastructure- service. 
[17] X. Yan, Q. Xue, L. Ma, and Y. Xu, "Driving-simulator-based test on the effectiveness of auditory red-light running vehicle warning system based on time-to-collision sensor," Sensors, vol. 14, no. 2, pp. 3631-3651, 2014.

[18] C. Olaverri-Monreal, P. Gomes, M. K. Silvéria, and M. Ferreira, "In-vehicle virtual traffic lights: a graphical user interface," in Proceedings of the 2012 7th Iberian Conference on Information Systems and Technologies (CISTI), pp. 1-6, 2012.

[19] OpenStreetMap, "Openstreetmap Statistics Openstreetmap stats report,” 2016, http://www.openstreetmap.org/stats/data_ stats.html.

[20] C. Biurrun-Quel, L. Serrano-Arriezu, and C. Olaverri-Monreal, "Microscopic driver-centric simulator: Linking Unity3d and SUMO," in Proceedings of the 5th World Conference on Information Systems and Technologies (WorldCist'17), vol. 569, pp. 851860, Springer, Madeira, Portugal, 2017.

[21] "Traas - TraCI as a Service" http://traas.sourceforge.net/cms/.

[22] "Geodatenviewer der Stadtvermessung Wien," 2017, https:// www.wien.gv.at/ma4ldatenviewer/public/.

[23] N. Oshiro, M. Matsushita, Y. Namikawa, and H. Ohnishi, "Fuel consumption and emission factors of carbon dioxide for motor vehicles," Civil Engineering Journal, vol. 43, no. 11, pp. 50-55, 2001.

[24] StatisticSolutions, "Statistic Solutions (M)Anova Analysis," 2017, http://www.statisticssolutions.com/manova-analysis-pairedsample-t-test/.

[25] "VSimRTI - Smart Mobility Simulation," 2017, https://www .dcaiti.tu-berlin.de/research/simulation/. 


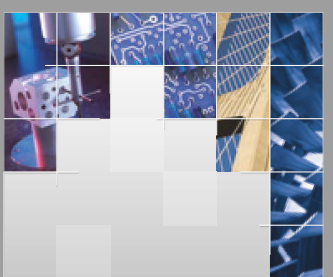

\section{Enfincering}
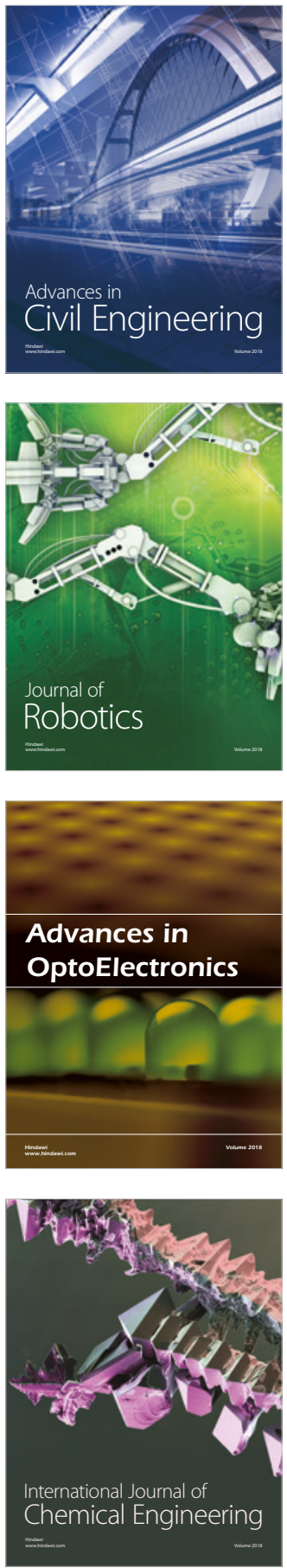

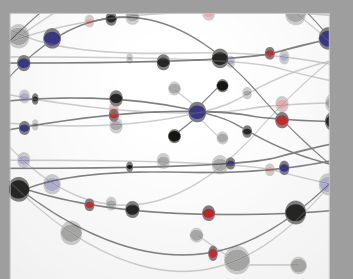

\section{Rotating \\ Machinery}

The Scientific World Journal

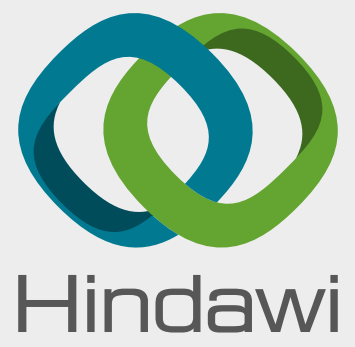

Submit your manuscripts at

www.hindawi.com
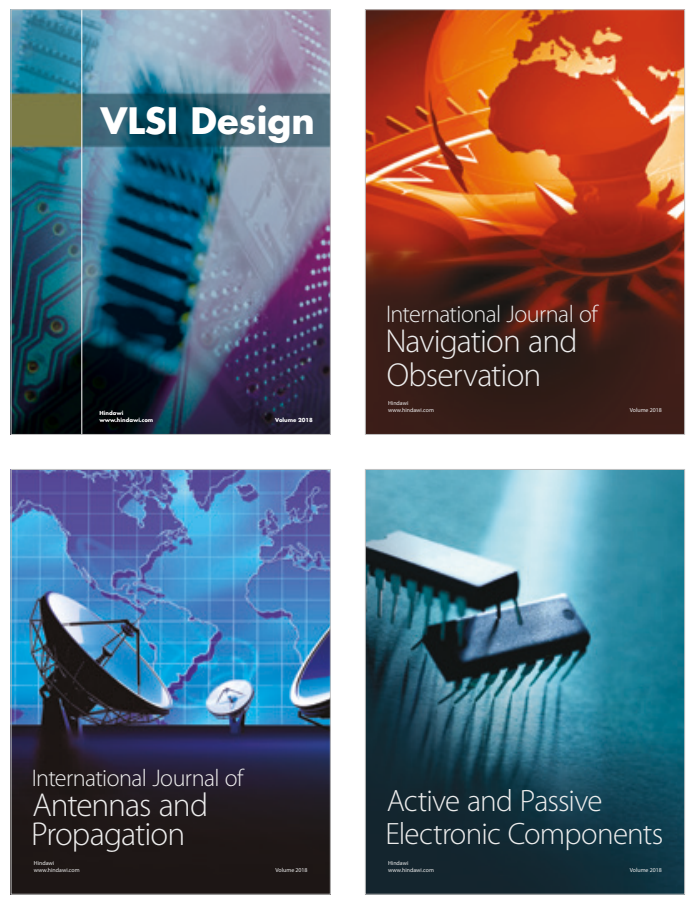
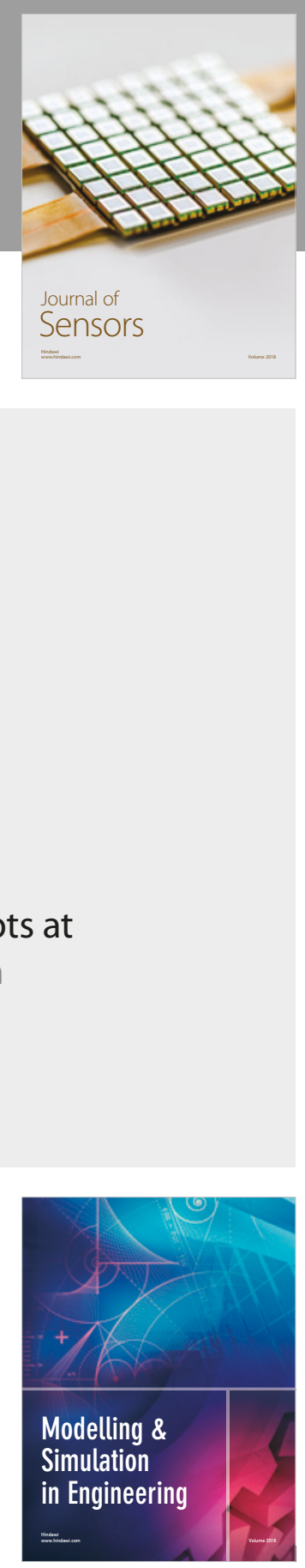

\section{Advances \\ Multimedia}
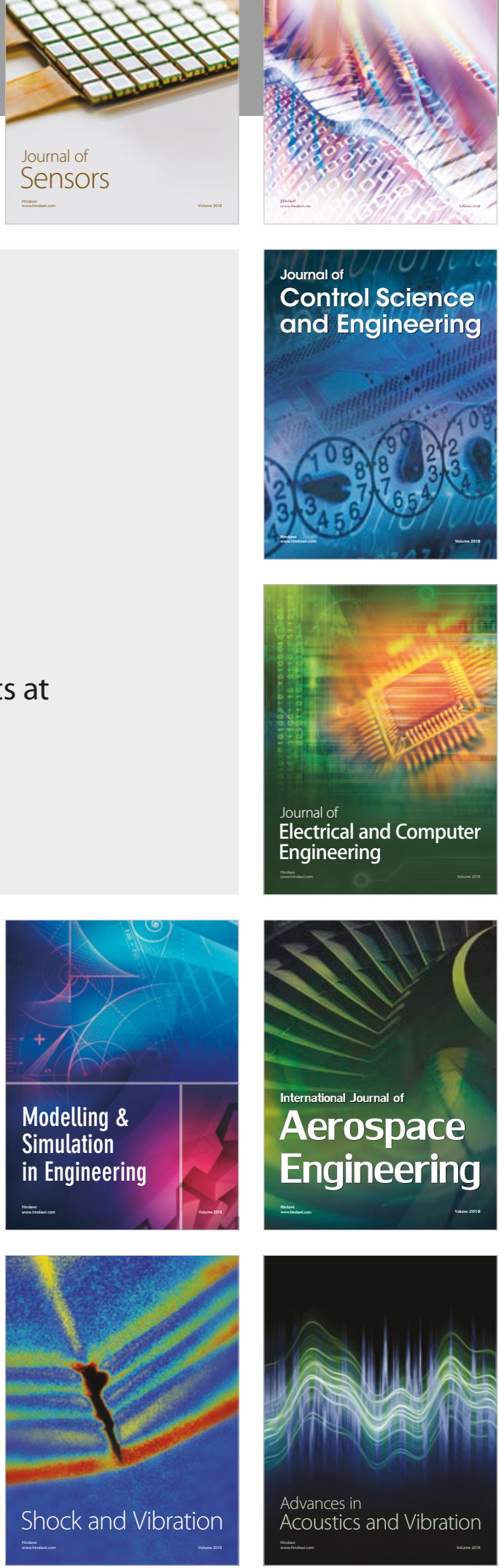